\title{
Pre- and postnatal imaging of Pai syndrome with spontaneous intrauterine closure of a frontal cephalocele
}

\author{
Tomas Dobrocky • Lukas Ebner • Benjamin Liniger • \\ Christian Weisstanner • Enno Stranzinger
}

Received: 24 April 2014 /Revised: 8 September 2014 / Accepted: 1 October 2014 /Published online: 31 October 2014

(C) Springer-Verlag Berlin Heidelberg 2014

\begin{abstract}
Pai syndrome is a rare congenital disorder characterized by cutaneous polyps of the face, pericallosal lipoma and median cleft lip. We report on a newborn girl with a variant of Pai syndrome presenting with all typical findings except a median cleft. In addition, fetal sonography and MRI showed the unique intrauterine evolution of a cephalocele into an atretic cephalocele.
\end{abstract}

Keywords Pai syndrome $\cdot$ Prenatal diagnosis $\cdot$ Magnetic resonance imaging $\cdot$ Ultrasound $\cdot$ Fetus $\cdot$ Neonate

\section{Introduction}

Nasal polyps, forehead and pericallosal lipoma, corpus callosum hypoplasia and cephalocele are known pathologies but have rarely been observed as a combination in a single patient and visualized pre- and postnatally. A rare syndrome encompassing those findings was first described in 1987 by Pai and colleagues [1] and is characterized by cutaneous polyps of the face and nasal mucosa, midline lipomas of the central nervous system and an associated median cleft lip.

T. Dobrocky $(\bowtie) \cdot$ L. Ebner $\cdot$ E. Stranzinger

Department of Interventional, Pediatric and Diagnostic Radiology, Inselspital University Hospital, University of Bern,

Freiburgstrasse 10, 3010 Bern, Switzerland

e-mail: tomas.dobrocky@insel.ch

B. Liniger

Department of Pediatric Surgery, Inselspital University Hospital, University of Bern, Bern, Switzerland

\section{Weisstanner}

Department of Diagnostic and Interventional Neuroradiology, Inselspital University Hospital, University of Bern,

Bern, Switzerland

\section{Case report}

We report on a 2-day-old near-term caucasian girl with a palpable round mass on the forehead and a pedunculated lesion protruding from the right nasal cavity. The girl was born at 37 weeks 1 day of gestation by secondary forceps after delay of delivery with fetal bradycardia. After stimulation and maskventilation she saturated well at room air. The APGAR scores recorded at 1,5 and $10 \mathrm{~min}$ after birth were 2, 8 and 10, and body weight was $3.07 \mathrm{~kg}$. After delivery the girl was transferred to the intensive care unit of our institution for observation.

Because ventriculomegaly was noted by an outside gynecologist on routine prenatal US at 20 weeks' gestation, she was referred to our center for further evaluation. Chorionic villus sampling showed a normal karyotype 46 XX. To confirm the fetal US finding and to rule out associated cerebral anomalies, fetal MRI was conducted at 21 weeks' gestation and again at 30 weeks' gestation using a 1.5 T Avanto scanner (Siemens Healthcare, Erlangen, Germany). The non-contrast MRI cranial protocol included the following: T1-weighted and ultrafast T2-weighted HASTE (half-Fourier acquisition single-shot turbo spin-echo) sequences and a T2-weighted gradient echo sequence (true fast imaging with steady-state free precession [trueFISP]) in all three planes; and diffusionweighted images (DWI) at $b=0, b=500, b=700$ and $b=$ $1,000 \mathrm{~s} / \mathrm{mm}^{2}$ in axial and coronal planes. The initial MRI showed a 7-mm midline osseous defect in the frontal bone with protruding cerebrospinal fluid (CSF) and no evidence of brain tissue, indicating that it was most likely a meningocele (Fig. 1). In addition, MRI showed a slightly T1-hyperintense and T2-hypointense lesion in the right nasal cavity measuring $4 \mathrm{~mm}$ in cross-sectional diameter. Further findings were normal according to gestational age; no sulcation abnormalities and no pathology of the ventricle system as described on sonography were present. No extracranial malformation was apparent on prenatal imaging. 
Fig. 1 Fetal MRI at 21 weeks' gestational age. a Sagittal T2weighted image (TrueFISP, repetition time/echo time $[\mathrm{TR} / \mathrm{TE}]$ $4.19 / 1.83 \mathrm{~ms}$ ) and (b) axial T2weighted image (HASTE, TR/TE $1,260 / 85 \mathrm{~ms}$ ) show a defect in the frontal bone and protruding meningeal sac filled with hyperintense liquid (arrow), a finding consistent with frontal cephalocele. HASTE half-Fourier acquisition single-shot turbo spinecho, TrueFISP true fast imaging with steady-state free precession
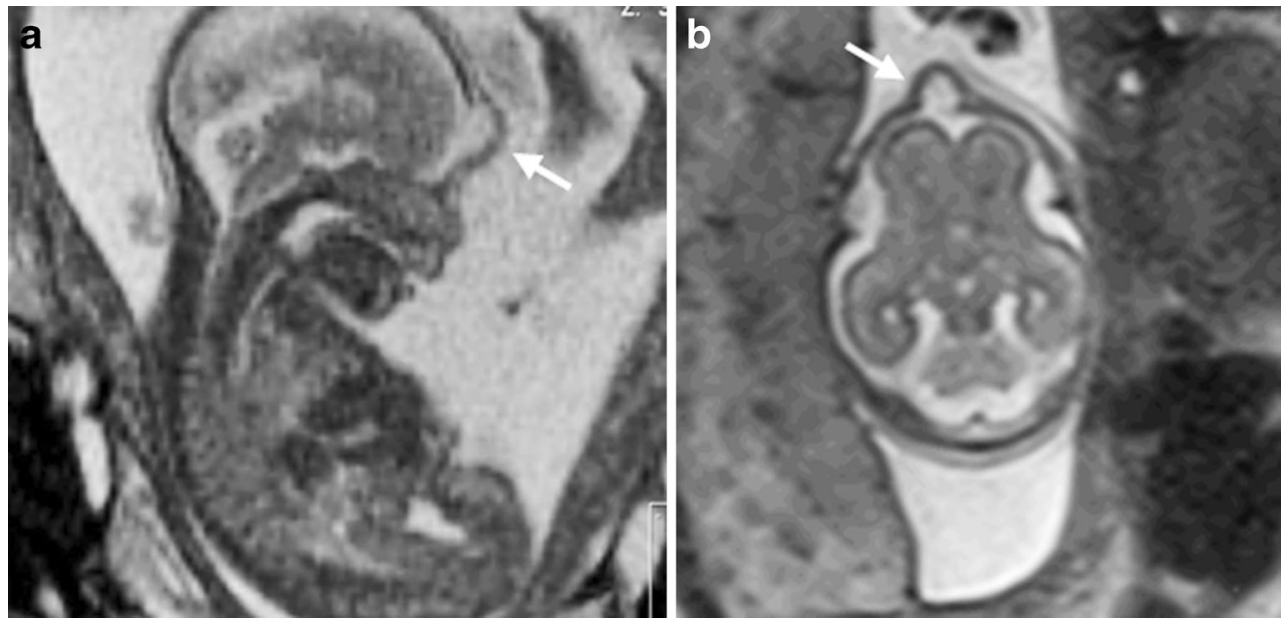

On control MRI performed at 30 weeks' gestation the previously observed frontal cephalocele showed apparent complete regression, with a residual gapping of the metopic suture and a small T1-hyperintense and T2hypointense outpouching overlying the frontal bone (Fig. 2). Retrospectively a T1-weighted coronal plane image shows a slender (width $2 \mathrm{~mm}$ ), hyperintense structure located in the interhemispheric fissure corresponding to the postnatally diagnosed pericallosal lipoma (Fig. 2). No change of the pre-existent appendage in the right nasal cavity was noted. The fetal brain parenchyma, the ventricle system, and the spine did not show any abnormalities.

On the second postpartum day transcranial US (Acuson S2000; Siemens, Erlangen, Germany) showed a hypoplastic corpus callosum, which could not be visualized in its posterior part, and a hyperechogenic 4-cm-long and 1-cm-thick longitudinal midline mass bordering the cranial part of corpus callosum, a finding suggestive of curvilinear pericallosal lipoma (Fig. 3). Further findings on sonography included hyperechogenic mass of the forehead (diameter $2.5 \mathrm{~cm}$, thickness $0.5 \mathrm{~cm}$ ) and a pedunculated tumor protruding from the nasal cavity $(1 \times 0.5 \times 0.5 \mathrm{~cm})$. No abnormalities were found on spinal sonography.

An MRI performed when the girl was 7 days old confirmed a hypoplastic corpus callosum with an adjacent interhemispheric lipoma, which was not evident on prenatal studies (Fig. 4). MRI confirmed a lesion, isointense to fat, in the nasal cavity with no connection to intracranial structures. A second lesion with similar intensity was localized in the forehead area, with a gapping of the
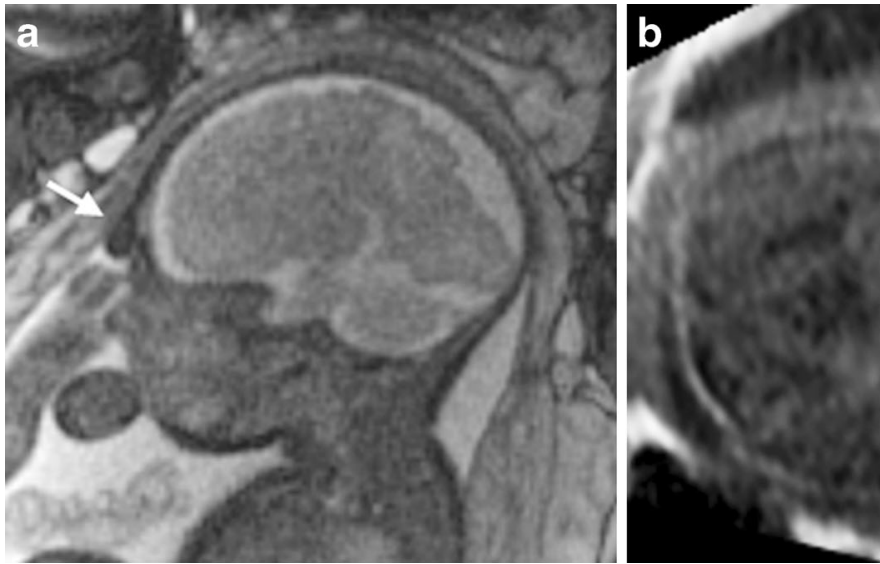

Fig. 2 Fetal MRI at 30 weeks' gestation. a Sagittal T2-weighted TrueFISP image (TR/TE 4.19/1.83 ms) and (b) axial T1-weighted FLASH-2D image (TR/TE 74/4.76 ms) show a hypointense (a) and a hyperintense (b) lesion in the forehead region (arrow), a finding consistent with lipoma. The pre-existent frontal cephalocele was no longer

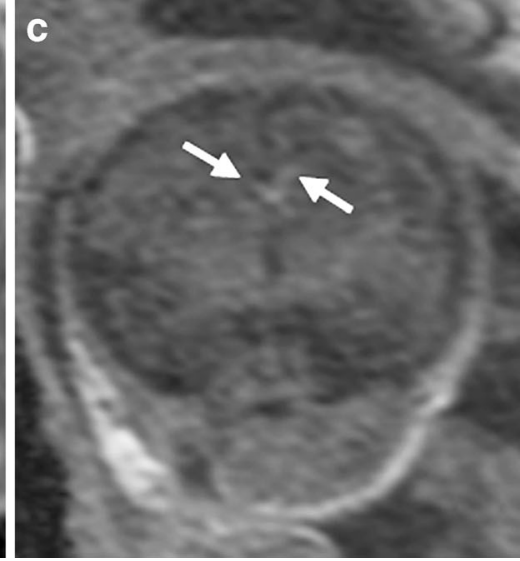

present, and residual gapping of the metopic suture was noted (not shown). c T1-weighted reconstructed coronal image retrospectively demonstrates a hyperintense lesion (width $2 \mathrm{~mm}$ ) in the interhemispheric fissure (arrows) that was not detected initially. FLASH-2D two-dimensional fast low-angle shot, $T R / T E$ repetition time/echo time 
Fig. 3 Transcranial US in a 2day-old girl. a Sagittal image and (b) coronal image acquired with a 9-MHz linear transducer show a hyperechogenic mass (arrows) in the interhemispheric fissure bordering the hypoplastic corpus callosum cranially, typical findings of pericallosal lipoma
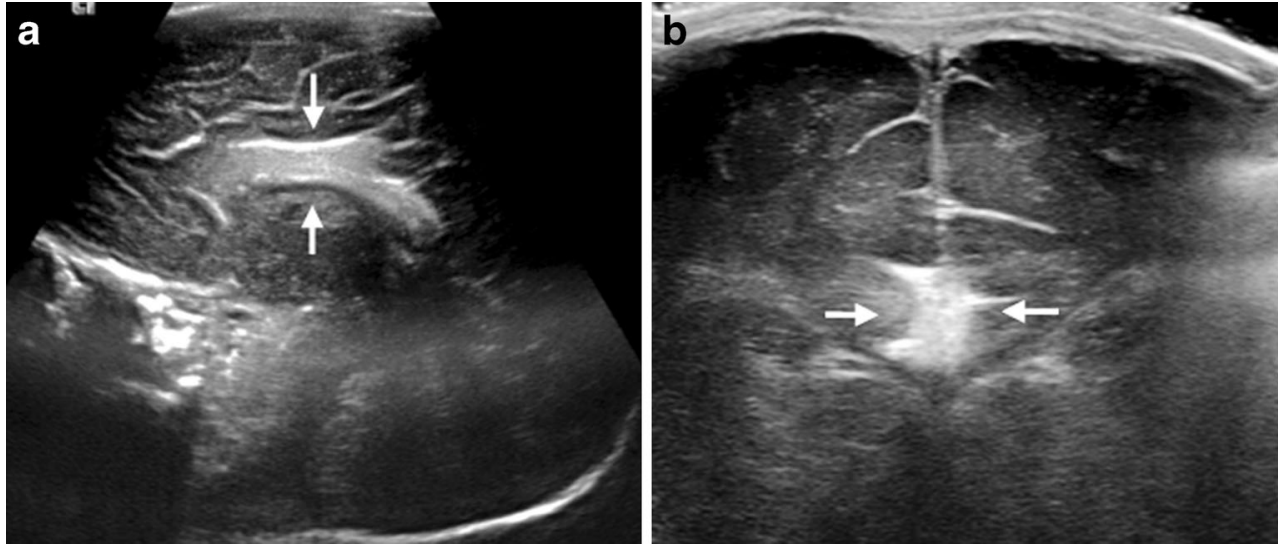

metopic suture (Fig. 4). No cleft of the maxillar or palate bone was detected. At discharge oxygen saturation at room air was $100 \%$ and there was a high-pitched wheezing originating at the right nasal introitus.

Three months later an endoscopic resection of the nasal appendage showed squamous cells, respiratory epithelium and underlying fibrous fatty tissue on histology, findings consistent with a cutaneous nasal polyp. When the girl was 18 months old an open resection of the frontal mass showed a focal gaping of the metopic suture and a funnel-shaped galea aponeurotica extending intracranially and attaching to the dura mater (Fig. 5). The two layers were separated and the gap in the metopic suture was covered with periosteum. Histology of the frontal mass showed a fibrous fatty tissue without presence of neural cells, consistent with lipoma. The girl showed appropriate psychomotor development (developmental quotient 95 , maximum 100 , norm $>85$ ) and a normal neuropediatric status at age $2 \frac{1}{2}$ years, the time of this report.

\section{Discussion}

In 1987 Pai et al. [1] described a syndrome characterized by cutaneous polyps of the face and nasal mucosa, midline lipoma of the central nervous system (pericallosal lipoma) and midline cleft lip. Our patient presented all symptoms as described by Pai except a midline cleft lip, which is not requisite for the diagnosis of Pai syndrome according to the modified diagnostic criteria published by Lederer et al. [2]. In the review by Lederer summarizing 36 patients with Pai syndrome, $20 \%$ did not present with a midline cleft lip [2].

The remarkable finding in our patient is the prenatally documented frontal cephalocele, with consecutive spontaneous intrauterine closure. Only one similar case report, by Ergun et al. [3], was found in the literature, and Ergun presented a patient with postnatal diagnosis of Pai syndrome and associated frontal cephalocele with overlying lipoma.

Cephaloceles represent skull defects with protrusion of intracranial structures, and according to Tortori-Donati and Rossi [4] these can be classified into four types: (1)
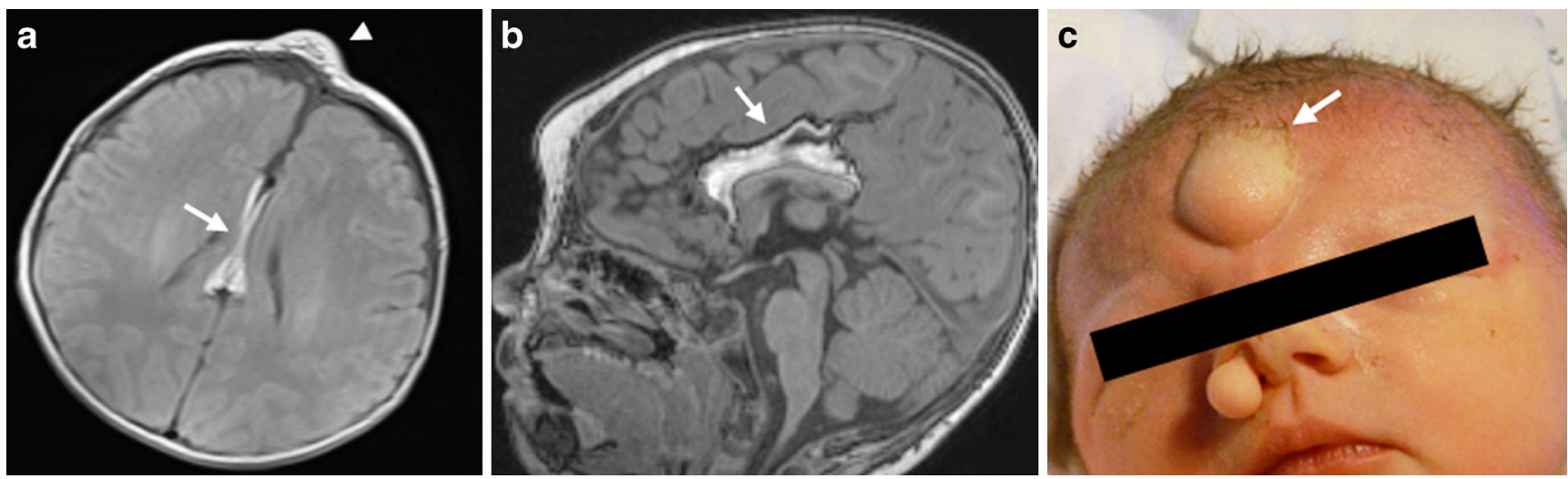

Fig. 4 Cranial MRI in the same girl at 7 days old. a Axial and (b) midsagittal T1-weighted images (TR/TE 1,500/13 ms) show a slender interhemispheric lipoma (arrows). There is a subcutaneous lesion, isointense to fat, in the forehead area (arrowhead in a) with gapping of the metopic suture in the same position as the cephalocele seen on first prenatal MRI at 20 weeks' gestation. c Clinical photograph of the girl's face shows a stalked lesion protruding from the right nasal cavity and a mass (arrows) in the forehead region. TR/TE repetition time/echo time 


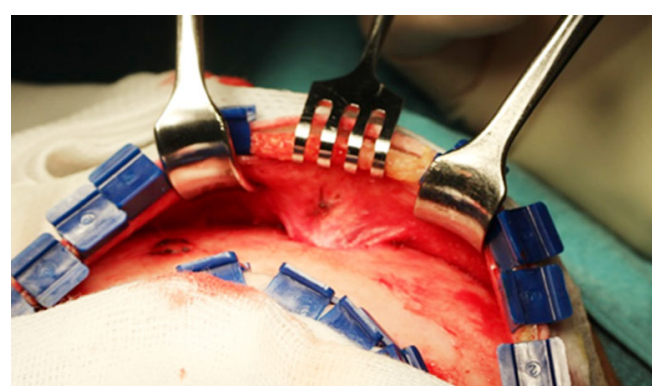

Fig. 5 Intraoperative image of the same girl at 18 months old shows gaping of the metopic suture and a funnel-shape galea aponeurotica extending intracranially and attaching to the dura

meningoceles containing CSF and lined by an epithelized meningeal wall; (2) meningoencephaloceles or encephaloceles that encompass additional brain tissue, (3) meningoencephalocystoceles, which are encephaloceles containing a portion of the ventricles; and (4) atretic cephaloceles, which represent a rudimentary form composed of small fibrolipomatous tissue. These pathologies predominantly occur in the midline and can further be subclassified based on their location as anterior or posterior. In general, posterior cephaloceles are more common than anterior lesions. The geographic distribution shows a much lower incidence of frontal cephaloceles in Europe and North America than in Southeast Asia. The ratio of occipital to frontal encephaloceles has been reported to be from 15:1 to 1.1:1, and in most series in the United States the ratio was above 5:1 [5]. The etiology of cephaloceles remains controversial, but two major theories exist. The first theory explains cephaloceles as neural tube closure defects, the second as postneurulation defects from failure in bony covering of the neural tube. The outcome in children with frontal defects is generally better; survival rates range $80-93 \%$ in those with frontal defects and $40-75 \%$ in those with posterior defects [4]. Furthermore, the size of the sac, protrusion of brain tissue and presence of hydrocephalus are predictors of the outcome.

The initial fetal MRI of our patient at 21 weeks' gestation demonstrated a frontal cephalocele, with spontaneous regression at follow-up at 30 weeks' gestation. On postnatal imaging there was a fat-isointense frontal lesion with a defect of the underlying calvarial bone in the region of pre-existent cephalocele and no evidence of a fibrous tract extending intracranially. Histology showed fibrolipomatous tissue with no cerebral tissue, findings consistent with an atretic cephalocele. Atretic cephaloceles are skin-covered midline subscalp masses also termed atypical meningoceles, rudimentary meningoceles, meningeal heterotopias or meningoceles manqué. These lesions contain varying amounts of dermal, meningeal, glial as well as fibrolipomatous tissue.

Spontaneous intrauterine regression of myelomeningoceles — another type of neural tube defect — has been reported [6].
However we are not aware of a case showing the spontaneous intrauterine closure of a cephalocele.

Concerning the diagnosis of pericallosal lipoma, because of its slender appearance and the image parameters used on fetal MRI (matrix $292 \times 512$ and slice thickness of $4 \mathrm{~mm}$ ), as well as minor fetal movements, the pathology was not well depicted on prenatal images. Even retrospectively it cannot be detected on fetal MR images performed at 21 weeks' gestation. However T1weighted images acquired at 30 weeks' gestation that were retrospectively reconstructed in the coronal plane (Fig. 2) show a slightly hyperintense lesion (width $2 \mathrm{~mm}$ ) in the interhemispheric fissure, corresponding to the postnatally diagnosed pericallosal lipoma.

Based on imaging, two types of pericallosal lipomas can be distinguished. The more frequent tubulo-nodular lipoma, which appears rounded or lobular and anteriorly situated, and the curvilinear lipoma, which is thin and posteriorly situated [7]. The tubulo-nodular type is often associated with additional intracranial pathologies, especially of the corpus callosum, and thus has a less favorable outcome [8].

Fetal MRI complements the information provided by obstetric sonography and is a necessary tool for an accurate diagnosis in fetal brain imaging, allowing more targeted counseling especially in Pai syndrome, which is associated with additional pathologies. In our case it seems of special interest to accurately classify the type of cephalocele and its contents because the outcome varies among entities. Furthermore, it seems relevant to check the size of the cephalocele because intrauterine volume increase might indicate a caesarean section to prevent fetal or maternal trauma during vaginal delivery.

Seven cases with Pai syndrome were reported as showing no neurological impairment, and normal psychomotor development [9]. Nonetheless antenatal counseling remains challenging, and termination of pregnancy in prenatally diagnosed Pai syndrome with anteriorly located pericallosal lipoma has been published by Blouet et al. [7]. Small lesions like slender pericallosal lipoma may be difficult to detect or even missed on prenatal imaging because of their size; nonetheless their characterization (location, size and extension) is important because outcomes vary.

In conclusion, our case presenting nasal polyps and forehead and pericallosal lipoma demonstrates a type of Pai syndrome, according to the modified diagnostic criteria published by Lederer et al. [2]. The unique finding in our case is the presence of an associated frontal cephalocele with spontaneous in utero closure. Different imaging modalities (US and MRI) at different time points may be necessary to establish the final diagnosis. 


\section{Conflicts of interest None}

\section{References}

1. Pai GS, Levkoff AH, Leithiser RE (1987) Median cleft of the upper lip associated with lipomas of the central nervous system and cutaneous polyps. Am J Med Genet 26:921-924

2. Lederer D, Wilson B, Lefesvre $P$ et al (2012) Atypical findings in three patients with Pai syndrome and literature review. Am J Med Genet A 158A:2899-2904

3. Ergün R, Okten AI, Bostanci U (1999) An unusual case of cephalocele associated with lipoma of corpus callosum. Pediatr Neurosurg 30:30-32

4. Tortori-Donati P, Rossi A (2005) Cephaloceles. In: Pediatric neuroradiology. Springer, Berlin, pp 72-86
5. Suwanwela C (1972) Geographical distribution of frontoethmoidal encephalomeningocele. Br J Prev Soc Med 26: 193-198

6. Klein O, Thompson D (2007) Spontaneous regression of lipomyelomeningocele associated with terminal syringomyelia in a child. Case report. J Neurosurg 107:244-247

7. Blouet M, Belloy F, Jeanne-pasquier C et al (2014) Pai syndrome: challenging prenatal diagnosis and management. Pediatr Radiol 44: $1184-1187$

8. Ickowitz V, Eurin D, Rypens F et al (2001) Prenatal diagnosis and postnatal follow-up of pericallosal lipoma: report of seven new cases. AJNR Am J Neuroradiol 22:767-772

9. Szeto C, Tewfik TL, Jewer D, Rideout A (2005) Pai syndrome (median cleft palate, cutaneous nasal polyp, and midline lipoma of the corpus callosum): a case report and literature review. Int J Pediatr Otorhi 69:1247-52 